\title{
A non-standard analysis approach to a constrained forced oscillator
}

\author{
DALIBOR PRAŽÁK \\ KUMBAKONAM R RAJAGOPAL \\ JAKUB SLAVÍK
}

\begin{abstract}
A non-autonomous oscillator with unilateral constraint is modeled using non-standard analysis. Existence and continuous dependence on initial conditions is obtained.
\end{abstract}

2010 Mathematics Subject Classification 34A09 (primary); 26E35, 34A12 (secondary)

Keywords: non-autonomous oscillator, second order ODEs, non-standard analysis, uniqueness of solutions

\section{Introduction}

Historically, the classical Newtonian mechanics has been closely connected with the development of Calculus and it still remains a rich source of problems to be formulated and solved in the language of differential and integro-differential equations. Perhaps one of the simplest examples of an important practical problem that is solved within the context of ordinary differential equations is the forced conservative oscillator

$$
m x^{\prime \prime}+F_{s}=f(t)
$$

of a mass $m>0$, with unknown displacement $x=x(t)$, driven by an external force $f(t)$ and a restoring (spring) force $F_{s}$. It is customary to close the system by a constitutive relation of the form

$$
F_{s}=h(x)
$$

with some reasonable function $h(x)$, as eg in the linear so-called Hooke's law $h(x)=k x$. This turns (1) into a second order ODE, a thoroughly studied mathematical object for which a well-developed theory of existence, uniqueness and qualitative properties of solutions is available. For $h(x)$ linear or piecewise affine, one can also express the solution explicitly in terms of quadratures and elementary functions. 
There, however, exist modifications both physically reasonable and interesting from point of view of applications, of the forced oscillator problem (1), for which the classical framework no longer suffices. It might be that the relationship between $x$ and $F_{s}$ does not assume the explicit functional form (2) (see Rajagopal [9] for a discussion of the relevance of implicit relationships between the displacement and spring force). A related possibility is that the motion is further restricted by some obstacles. Both these clearly go beyond the classical setting: globally smooth solutions cannot exist.

Implicit relationships between $x$ and $F_{S}$ can be studied via the general theory of differential inclusions, see Filippov [2], and the so-called non-smooth dynamical systems in Kunze [4]. For particular simple examples of oscillators with obstacles, it is possible to "patch" the global solution by imposing bounce conditions, see eg Marò [7], Kunze and Ortega [5] and Kunze and Ortega [6]. In the present paper, we propose an alternative way. Instead of providing an ad hoc generalized mathematical framework, we systematically enlarge our universe by working within the context of non-standard analysis (NSA). In virtue of the well-known principle of transfer, the whole classical ODE theory remains to our disposal and the new universe has the best of both worlds: it remains conceptually simple, yet rich enough to capture a number of non-classical phenomena.

In particular, we focus here on the problem of oscillator whose motion is restricted to $x \leq 0$ by an inextensible string; perhaps more aptly, we will think of an infinitely tough wall located at $x=0$. The description of this problem in the framework of NSA suggests the following: we model the wall as a spring with infinitely large stiffness. More precisely, we extend the equation (1), valid for $x<0$, by $m x^{\prime \prime}+L x=f(t)$ for $x>0$, where $L>0$ is an infinitely large positive number. The question of existence of solution is then solved in one shot, transferring the classical Picard's theorem. It is also easy to see that the solution does what we expect: the mass is bounced back immediately with opposite velocity (cf Proposition 2 below).

Our main focus is the problem of uniqueness, or perhaps more precisely, of macroscopic stability. The question is: does infinitely small perturbation of initial data only lead to infinitely small deviation of solution? Note that this does not follow from Picard's theorem: there is an infinitely large Lipschitz constant $L>0$ in our equation, hence the general theory allows for a factor $e^{L t}$ growth of disturbances, which is infinitely large for standard $t>0$. A second natural and related question reads: is the macroscopic (ie modulo infinitely small error) behavior of solution independent of the particular choice of the infinitely large constant $L>0$ ? In the main result of the paper (Theorem 2) we are able answer both of these questions affirmatively. 
We also note that the "standard" way to treat the problem of inextensible string would eventually lead to a sequence of approximating problems, where $L$ is replaced with standard $L_{n} \rightarrow+\infty$. The expected result is that the approximating solutions $x_{n}$ converge to some $x$, which is a continuous (even smooth if $x<0$ ) function, cf Yuan et al [12]. And this is precisely what we arrive at in our last Theorem 3, simply by transferring both our original equation and the result of Theorem 2 to the standard world. The theorems are logically equivalent, but one can argue that the language of NSA is better suited both for the physical description of the problem, and the mathematical theorem and its proof.

Two points seem worth emphasizing. Any application of the NSA means that some initial theory is taken into an extended universe, giving rise to new concepts and new methods of reasoning. Our initial theory is, however, highly nontrivial: essentially what we need is to transfer the classical ODE theory for Lipschitz nonlinearities, Riemann integral, Picard's theorem and also the variation of constants formula. What we gain, in turn, is a conceptually simple, yet highly powerful framework, in which problems involving differential inclusions, weak convergence and estimates of oscillations are tractable by truly elementary analytical means.

Secondly, we would like to pinpoint what in our opinion is the central issue of the paper. It is the question as to what the oscillator does when the constraint at $x=0$ is reached, but the external force $f(t)$ is positive. In particular, what will happen if the velocity is zero at such a moment? This is precisely the point where the classical description fails most markedly, possibly also the situation that even baffles our intuition as to what the dynamics ought to be. To solve the problem, we impose an additional structural assumption on the right-hand side $f(t)$, $\mathrm{cf}(9)$ below. We are then able to show that although the system can switch between the two regimes $x>0$ and $x<0$ infinitely (more precisely, hyper-finitely) many times, only an infinitesimal increase of energy is possible as long as $f(t) \geq 0$.

The paper is organized as follows: the problem under study is described in Section 2 and the main three theorems are formulated. In Section 3, we outline the required background from NSA. In particular, we explain the theory within which we work and prove Theorem 1 in detail. We also show how Theorem 3 follows from Theorem 2.

Section 4 forms the main core of the paper and it deals with the crucial problem: how will the solution with initial conditions infinitely close to the wall $x=0$ behave?

Section 5 is devoted to the proof of the main Theorem 2. In Section 6 we prove three auxiliary lemmata on "integration" of various type difference inequalities, which are instrumental in the proof of the key Proposition 4. 


\section{Problem formulation and main theorems.}

We study the behavior of a standard oscillator with external forcing, whose motion is restricted to $x \leq 0$ by an infinitely elastic wall:

$$
\begin{aligned}
m x^{\prime \prime}+s(x) & =f(t), \quad t \in I \\
x(0) & =x_{0}, \\
x^{\prime}(0) & =v_{0},
\end{aligned}
$$

with

$$
s(x)=\left\{\begin{array}{l}
h(x), \quad x<0 \\
L x, \quad x \geq 0 .
\end{array}\right.
$$

We $\operatorname{set}^{1} I={ }^{*}[0, T]$, where $T>0$ is a standard positive number; the restoring force is given by a standard function $h(x) \in C_{\text {loc }}^{1}((-\infty, 0])$. It is convenient and without loss of generality to assume that $h(0)=0$ and $h(x)$ is globally (standard) Lipschitz. Finally $L>0$, modeling the response of the wall, is infinitely large positive number.

The initial data $x_{0}, v_{0}$ are hyperreal limited, with $x_{0} \succsim 0$. External force is a standard continuous function and for the sake of notational simplicity we henceforth set the mass $m$ equal to 1 .

Theorem 1 The problem (3-6) has a unique solution on I.

Proof This follows immediately by transferring the Picard's existence theorem; see Section 3 for more details.

The energy is defined in the usual manner:

$$
E(t)= \begin{cases}\frac{1}{2}\left(x^{\prime}\right)^{2}(t)+\frac{L}{2} x^{2}(t), & x(t) \geq 0, \\ \frac{1}{2}\left(x^{\prime}\right)^{2}(t)+H(x(t)), & x(t) \leq 0,\end{cases}
$$

where $H(x)=\int_{0}^{x} h(\xi) d \xi$ is primitive to $h(x)$. It is easy to show that the energy remains limited, provided the data are so. Here and in what follows, $c>0$ stands for a generic standard constant.

Lemma 1 One has $E(t) \leq c$ for any $t \in I$, where $c$ is a standard constant depending on the data $x_{0}, v_{0}, f(t)$ and $T>0$. Moreover, if $x(t)>0$, then $x(t) \leq c L^{-1 / 2} \approx 0$.

\footnotetext{
${ }^{1}$ See next Section for definition of $*, \approx$, etc.
} 
Proof Multiply the equation by $x^{\prime}$. We have

$$
\frac{d}{d t} E(t)=x^{\prime}(t) f(t) \leq \frac{1}{2}\left(x^{\prime}(t)\right)^{2}+\frac{1}{2}(f(t))^{2} \leq E(t)+c
$$

and estimate $E(t)$ using transferred version of the Gronwall's lemma. Then second assertion follows since for $x>0$, (7) implies $|x| \leq(2 E / L)^{1 / 2}$.

From this point of view, the existence part of the theory seems satisfactory. We also note that $\left|x^{\prime}\right| \leq c$, ie the solutions are globally Lipschitz. But there is a serious problem lurking behind the uniqueness. Note that by standard argument, we obtain the following.

Lemma 2 Let $x(t), \tilde{x}(t)$ be two solutions that are negative on some $\left[t_{0}, t_{1}\right] \subset[0, T]$. Then

$$
|x(t)-\tilde{x}(t)|+\left|x^{\prime}(t)-\tilde{x}^{\prime}(t)\right| \leq c\left(\left|x\left(t_{0}\right)-\tilde{x}\left(t_{0}\right)\right|+\left|x^{\prime}\left(t_{0}\right)-\tilde{x}^{\prime}\left(t_{0}\right)\right|\right) \quad \forall t \in\left[t_{0}, t_{1}\right]
$$

where $c$ is a standard constant only depending on the Lipschitz constant of $h(x)$.

Proof In the usual manner, the equation can be rewritten as

$$
\begin{aligned}
x^{\prime} & =y \\
y^{\prime} & =f(t)-h(x)
\end{aligned}
$$

where the right-hand side is Lipschitz with respect to $x, y$. The conclusion follows by Gronwall's lemma.

This lemma implies in particular what we would call a macroscopic stability: if the initial data are infinitely close at $t=t_{0}$, the solutions remain so for all $t \in\left[t_{0}, t_{1}\right]$. But this argument cannot be extended to cover the situation where possibly $x(t)$ or $\tilde{x}(t)$ becomes positive, since then $h(x)$ must be replaced by $s(x)$ and the Lipschitz constant of the right-hand side becomes infinitely large; cf (6).

Indeed, the equation is not in the above sense stable with respect to initial conditions. Assume that $f(t) \equiv 0, v_{0}=0$ and $x_{0}=L^{-1 / 2}$. It follows from Lemma 3 below that for $t_{1}=\pi L^{-1 / 2} / 2, x\left(t_{1}\right)=0$ and $x^{\prime}\left(t_{1}\right)=-1$, and thus $x(t)=\left(t_{1}-t\right)$ for $t \geq t_{1}$. Such a solution deviates appreciably from the equilibrium $x \equiv 0$, though it has an infinitely close initial condition. Note also that the velocity of the former solution has a macroscopic jump, more precisely the function $t \rightarrow \mathrm{st} x^{\prime}\left({ }^{*} t\right)$ is not continuous at $t=0$.

On the other hand, it is reasonable to expect that two solutions arriving at the wall from the outside, with infinitely close distances and velocities, will remain so after colliding with it. Even more, the value of $L$ should not play any role, as long as it is 
infinitely large. However, it also turns out that the right-hand side $f(t)$ cannot be too wild - additional structural assumption on the right-hand side seems necessary - and we henceforth require:

$$
f(t) \text { is a standard } C^{1} \text { function and if } f\left(t_{0}\right)=0 \text { for some } t_{0} \in[0, T],
$$$$
\text { then either } f_{+}^{\prime}\left(t_{0}\right) \neq 0 \text { or } f \equiv 0 \text { on some right neighbourhood of } t_{0} \text {. }
$$

The main result of our paper is the following theorem.

Theorem 2 Let $x(t)$ be a solution to (3-6). Let $f(t)$ satisfy (9) and let $\tilde{x}(t)$ be another solution with possibly different $\tilde{L} \approx \infty$ in $(6)$ such that $\tilde{x}(0) \approx x_{0}$ and $\tilde{x}^{\prime}(0) \approx v_{0}$. Then $x(t) \approx \tilde{x}(t)$ for all $t \in I$; and in the regime where $x(t)$ and $\tilde{x}(t)<0$ there holds $x^{\prime}(t) \approx \tilde{x}^{\prime}(t)$.

Our result can also be translated (more precisely, transferred) to the standard language; note that this also requires that the equation being studied must be reformulated.

Theorem 3 Let $x_{n}(t)$ be a sequence of solutions to (3-6) in a standard setting with $L=L_{n}$ being standard numbers converging to $+\infty$, subject to initial conditions $x_{0}, v_{0} \in \mathbb{R}, x_{0}<0$. Then $x_{n}(t)$ converge to some $x(t)$ uniformly in $[0, T]$. The mapping $\left(x_{0}, v_{0}\right) \rightarrow x(t)$ is continuous and $\left(x_{0}, v_{0}\right) \rightarrow x^{\prime}(t)$ is continuous at points where $x(t)<0$. Moreover, the function $x(t)$ solves the standard equation (3), (6) $)_{1}$ whenever $x(t)<0$.

\section{A brief review of NSA}

In this section we offer a brief and rather informal review of NSA. For a more exhaustive and detailed treatment we refer the reader to Hurd and Loeb [3] or Väth [11].

Let $S$ be a set and let $S_{0}=S$ and $S_{n+1}=S_{0} \cup \mathcal{P}\left(S_{n}\right)$. Then the superstructure of $S$ is defined by

$$
\widehat{S}=\bigcup_{n=1}^{\infty} S_{n} .
$$

The elements of $S$ are called atoms and the elements of $\widehat{S}$ that are not atoms are called entities.

Observe that the superstructure is large enough to contain practically every mathematical object we may be interested in, specially all the functions $f: X \rightarrow \mathbb{R}$ if $X$ and $\mathbb{R}$ are entities in $\widehat{S}$. 
The basic concept of NSA is the construction of a superstructure $\widehat{T}$ and of a map $*: \widehat{S} \rightarrow \widehat{T}$, the so-called elementary embedding, that serve as an abstract idealization of the superstructure $\widehat{S}$ and a transition map between the original superstructure and the idealized one. Although the actual construction of the superstructure $\widehat{T}$ and the elementary embedding $*$ can be achieved by different means (eg by abstract model theory, by working with ultrafilters or by axiomatization, cf Benci et al [1] or Nelson [8]), the particular method by which we obtain the elementary embedding plays no role in the applications.

Let us write $\widehat{* S}$ instead of $\widehat{T}$ and ${ }^{*} A$ instead of $*(A)$ and let $*: \widehat{S} \rightarrow \widehat{* S}$ be an elementary embedding.

Let $\alpha$ be a first order formula with constants in $\widehat{S}$. Then $\alpha$ is called transitively bounded if the scope of any quantifier is explicitly bounded, eg $(\forall x \in[0,1])(\exists y \in[0,1])\left[y^{2}=x\right]$. Obviously, any formula of the usual mathematical practice can be written in this form. The $*$-transform of $\alpha$ is the formula ${ }^{*} \alpha$ where we replace any constant $c$ by ${ }^{*} c$.

The following proposition, which will be referred to as "the transfer principle" rather than Theorem 4, is essential in NSA and justifies the transition from the original superstructure $\widehat{S}$ to the idealized superstructure $\widehat{* S}$. At the same time it ensures that all the tools we have in the original superstructure work in the idealization as well.

Theorem 4 (Transfer principle [11, Theorem 3.7]) Let $\alpha$ be a transitively bounded formula with constants in $\widehat{S}$. Then $\alpha$ holds true if and only ${ }^{*} \alpha$ holds true.

We emphasize that the elementary embedding $*: \widehat{S} \rightarrow \widehat{*}$ is not onto unless the set $S$ is finite, in which case the transition to $\widehat{* S}$ does not bring anything new. If an element is in the range of $*$, we call it a standard element. The elements of standard sets are called internal and the elements which are not internal are called external. In other words, $b$ is standard if $b={ }^{*} a$ for some $a \in \widehat{S}$ and $b$ is internal if $b \in{ }^{*} A$ for some entity $A \in \widehat{S}$.

We may think of the internal elements as the elements of $\widehat{* S}$ which can be described by the language of the original superstructure $\widehat{S}$. This can be made precise by the so-called internal definition principle but will not be needed in the rest of the paper. We only remark that the set of all internal entities is closed under finite set operations such as unions, intersections and subtractions (cf Väth [11, Theorem 3.19]) and the following characterization holds.

Theorem 5 [11, Theorem 3.21] Let $A, B \in \widehat{S}$ be entities and let $B^{A}$ denote the set of all functions $f: A \rightarrow B$. Then

$$
{ }^{*}\left(B^{A}\right)=\left\{f:{ }^{*} A \rightarrow{ }^{*} B ; f \text { is internal }\right\} .
$$


From now on, we assume that $\mathbb{R}$ is an entity in $\widehat{S}$. From the transfer principle and Theorem 5 we see that there exists a standard (and therefore internal) function ${ }^{*}|\cdot|:{ }^{*} \mathbb{R} \rightarrow{ }^{*} \mathbb{R}$ that satisfies all the properties of the standard modulus function, such as the non-negativity or subadditivity. This concept can actually be applied to all functions and relations on $\mathbb{R}$, eg the function sin or the ordering $\leq$. In the following we will drop the symbol * for all the standard functions and relations defined on ${ }^{*} \mathbb{R}$, eg we will write $|r|$ instead of ${ }^{*}|r|$ and $r \leq q$ instead of $r^{*} \leq s$, where $r, s \in{ }^{*} \mathbb{R}$. We will also identify the real numbers with their hyperreal counterpart, eg we will simply write 1 instead of ${ }^{*} 1$. Similarly for $a, b \in{ }^{*} \mathbb{R}$ we will use the notation

$$
[a, b]=\left\{x \in{ }^{*} \mathbb{R} ; a \leq x \leq b\right\}, \quad(a, b)=\left\{x \in{ }^{*} \mathbb{R} ; a<x<b\right\} .
$$

Observe that in view of the above we have $\left[{ }^{*} a,{ }^{*} b\right]={ }^{*}[a, b]$ for $a, b \in \mathbb{R}$ and these sets are internal.

We will call the elements of $* \mathbb{R}$ (or ${ }^{*} \mathbb{N}$ ) hyperreal (or hypernatural) numbers. A hyperreal number $r \in{ }^{*} \mathbb{R}$ will be called limited if there exists $n \in \mathbb{N}$ such that $|r|<{ }^{*} n$, unlimited if $|t|>{ }^{*} n$ for every $n \in \mathbb{N}$, infinitesimal if $|t|<{ }^{*}\left(n^{-1}\right)$ for every $n \in \mathbb{N}$. The key point is that the sets of all unlimited and infinitesimal numbers are not empty, cf Väth [11, Corollary 5.10].

By $a \approx b$ we mean that $a$ is infinitely close to $b$, ie $|a-b|$ is infinitesimal. Symbol $a \succsim b$ means that $a<b$ and $a \not b$. With a slight abuse of notation, the symbol $b \approx \infty$ means that $b$ is unlimited positive. Observe that the relation $\approx$ is not transitive anytime $\infty$ is involved.

By st $x$ we denote the standard part of a limited number $x \in{ }^{*} \mathbb{R}$, ie the uniquely defined real number $r \in \mathbb{R}$ such that ${ }^{*} r \approx x$. For $A \subset{ }^{*} \mathbb{R}$, we define st $A=\{$ st $a ; a \in A\}$.

An entity $A \in \widehat{*} S$ will be called hyperfinite if there exists some $N \in{ }^{*} \mathbb{N}$ and an internal bijection $f:\left\{n \in{ }^{*} \mathbb{N} ; n \leq N\right\} \rightarrow A$.

Let $f:{ }^{*} \mathbb{R} \rightarrow{ }^{*} \mathbb{R}$ be a function. The domain ${ }^{*} \mathbb{R}$ is chosen only for simplicity as all of the discussed features have an obvious analogue for suitable subsets of ${ }^{*} \mathbb{R}$. We say that $f$ is $*$-continuous in $x \in{ }^{*} \mathbb{R}$, if the function satisfies the transferred definition of continuity, namely

$\left(\forall \varepsilon \in{ }^{*} \mathbb{R}\right)\left(\exists \delta \in{ }^{*} \mathbb{R}\right)\left(\forall x \in B\left(x_{0}, \delta\right) \backslash\left\{x_{0}\right\}\right)\left[\varepsilon>0 \vee \delta>0 \Rightarrow\left|f(x)-f\left(x_{0}\right)\right|<\varepsilon\right]$.

We say that $f$ is $S$-continuous in $x_{0} \in{ }^{*} \mathbb{R}$ if $x \approx x_{0}$ implies $f(x) \approx f\left(x_{0}\right)$ with $x \in{ }^{*} \mathbb{R}$.

To relate the results obtained in the nonstandard idealization, ie the superstructure $\widehat{* S}$, to the standard superstructure $\widehat{S}$, we will use the following theorem. 
Theorem 6 (Hurd and Loeb [3, Exercise III.3.17]) Let $[a, b] \subseteq \mathbb{R}$ and let $f:{ }^{*}[a, b] \rightarrow$ $\mathbb{R}$ be an internal $S$-continuous function such that $f\left({ }^{*} x\right)$ is limited for all $x \in[a, b]$. Let $g:[a, b] \rightarrow \mathbb{R}$ be defined by $g(x)=\operatorname{st} f(x)$. Then $g$ is continuous on $[a, b]$.

Next we will discuss the calculus with internal $*$-continuous functions. It is quite common in the NSA-related research concerning differential equations to discretize the time or the space with an infinite precision and treat the differential equation as a difference equation with infinitesimal steps. We will use a different approach and work with the transferred version of the standard derivative and Riemann integral. However, this is only possible since we restrict ourselves to standard continuously differentiable right-hand sides of the equation (3). If we were to consider less regular right-hand sides, eg measures, a more refined approach would be required.

Let $D: C^{1}(\mathbb{R}) \rightarrow C(\mathbb{R})$ be an operator defined by

$$
(D(f))(x)=\frac{d}{d x} f(x), \quad x \in \mathbb{R} .
$$

Then the transfer principle allows us to work with the operator ${ }^{*} D:{ }^{*}\left(C^{1}(\mathbb{R})\right) \rightarrow{ }^{*}(C(\mathbb{R}))$ which we will call the $*$-derivative. The operator ${ }^{*} D$ satisfies the same properties as the operator $D$, specially ${ }^{*} D$ is linear and all the differentiation rules still apply. The transfer principle and Theorem 5 also shows that ${ }^{*}\left(C^{1}(\mathbb{R})\right)$ is the set of all the $*$-continuous internal functions defined on ${ }^{*} \mathbb{R}$ with $*$-continuous $*$-derivative.

Similarly, let $a, b \in \mathbb{R}$ and let $I_{a}^{b}: C([a, b]) \rightarrow \mathbb{R}$ denote the Riemann integral

$$
I_{a}^{b}(f)=\int_{a}^{b} f(s) d s
$$

Then by the transfer principle for every $a, b \in{ }^{*} \mathbb{R}$ there exists an operator

$$
{ }^{*} I_{a}^{b}:\left\{f:[a, b] \rightarrow{ }^{*} \mathbb{R} ; f \text { internal and } * \text {-continuous }\right\} \rightarrow{ }^{*} \mathbb{R}
$$

with properties matching the ones of the Riemann integral.

With another slight abuse of notation, we will not distinguish between the "standard" versions of the derivative and the integral and the transferred versions unless explicitly mentioned and we will use the standard notation.

Now we are ready to prove Theorem 1 and Theorem 3. Before the proofs, let us precisely state the definition of the solution to the problem (3-5), although it is clearly just a transferred version of the standard definition.

Definition An internal function $x:(a, b) \rightarrow{ }^{*} \mathbb{R}$, where $a, b \in{ }^{*} \mathbb{R}$ and ${ }^{*}[0, T] \subset(a, b)$, is a solution of the problem (3-5) if $x$ is twice $*$-differentiable on $(a, b)$, solves the equation (3) on $(a, b)$ and satisfies the initial conditions (4) and (5). 
Proof of Theorem 1 Observe that function $s(x)$ defined in (6) is globally Lipschitz continuous in nonstandard sense (with Lipschitz constant $L$ ). The existence of unique global solution then follows by transfer of the corresponding result from the classical ODE theory. Note that the solution operator $\left(t, x_{0}, v_{0}\right) \mapsto\left(x(t), x^{\prime}(t)\right)$ is internal, $*$-continuous function.

Proposition 1 Let $X(t)$ be a $*$-differentiable solution to

$$
X^{\prime}=F(t, X)
$$

on some standard interval $I={ }^{*}[0, T]$, where $F:{ }^{*} \mathbb{R}^{1+n} \rightarrow{ }^{*} \mathbb{R}^{n}$ is $*$-continuous. Assume that $J \subset I$ is an interval and $Q \subset \mathbb{R}^{n+1}$ a set such that graph $\left.X\right|_{J} \subset Q$.

1. If $F(t, X)$ is limited on $Q$, then $X(t)$ is $S$-continuous on $J$.

2. If moreover $J, Q$ are standard and $F(t, X)$ is standard and continuous on $Q$, then st $X$ is a classical solution to $X^{\prime}=\widetilde{F}(t, X)$ on $J$, where $F={ }^{*} \widetilde{F}$.

Proof 1 . For any fixed component $i=1, \ldots, n$ we get by transfer of the Lagrange theorem

$$
X_{i}(t+h)-X_{i}(t)=F_{i}(\tau, X(\tau)) h
$$

for any $t, t+h$ in $I$, where $\tau \in[t, t+h]$. If $|F| \leq M$ with some standard $M$, then clearly $X(t)$ is $M$-Lipschitz and in particular S-continuous.

2. From the first part we already know that $X(t)$ is S-continuous, hence st $X(t)$ is a well-defined continuous function. Assume now that $t, t+h \in J$ are standard. Applying the standard part on (10) leads to

$$
\text { st } X_{i}(t+h)-\text { st } X_{i}(t)=\text { st } F_{i}(\tau, X(\tau)) h .
$$

But also, thanks to the assumptions on $F$, we have

$$
\text { st } F(\tau, X(\tau))=\widetilde{F}(\text { st } \tau, \text { st } X(\tau)),
$$

hence by a classical limit argument we obtain

$$
\left(\text { st } X_{i}\right)^{\prime}(t)=\widetilde{F}_{i}(t, \text { st } X(t)) .
$$

Theorem 7 (Robinson [10, Theorem 4.2.1]) Let $(X, d)$ be a metric space and let $x \in X$. Let $\left\{x_{n}\right\}$ be a sequence in $X$. Then $x_{n} \rightarrow x$ in $X$ if and only if $d\left({ }^{*} x_{N},{ }^{*} x\right) \approx 0$ for every $N \in{ }^{*} \mathbb{N}$ unlimited. 
Proof of Theorem 3 Let $L \approx \infty$ be arbitrary and let $x_{L}$ be a solution to (3-6) from Theorem 1. Denote $x(t)=$ st $x_{L}(t)$ for $t \in[0, T]$. From Theorem 2 it follows that $x(t)$ does not depend on the particular choice of $L$. Moreover, the solution operator $\left(t, x_{0}, v_{0}\right) \mapsto x_{L}(t)$ is $S$-continuous, as is $\left(t, x_{0}, v_{0}\right) \mapsto x_{L}^{\prime}(t)$ if $x(t) \succsim 0$. Hence the assertion about continuity of $\left(x_{0}, v_{0}\right) \mapsto$ st $x(t)=x(t)$ and $\left(x_{0}, v_{0}\right) \mapsto$ st $x^{\prime}(t)$ follows from Theorem 6 .

To prove that $x(t)$ is a uniform limit of solutions to approximating problems, let $L_{n} \rightarrow \infty$ be a sequence of real numbers and $x_{n}(t)$ solutions of (3-6) posed in the standard setting with $L_{n}$ instead of $L$. Let $(X, d)$ be the space of continuous functions on $[0, T]$ with a metric $d$ induced by the supremum norm, ie with the topology of uniform convergence. Then by the transfer principle and Theorem 5 we have

$$
\begin{gathered}
{ }^{*} X=\left\{\tilde{x}:{ }^{*}[0, T] \rightarrow{ }^{*} \mathbb{R} ; \tilde{x} \text { is internal and } * \text {-continuous }\right\} \\
d(f, g)=\sup \left\{|f(t)-g(t)| ; t \in{ }^{*}[0, T]\right\} \quad \text { for } f, g \in{ }^{*} X .
\end{gathered}
$$

The supremum is well defined as the functions are $*$-continuous on a $*$-closed interval. Let $N \in{ }^{*} \mathbb{N}, N \approx \infty$ be arbitrary. Then using Theorem 2 we get ${ }^{*} x(t) \approx x_{N}(t)$ for $t \in{ }^{*}[0, T]$ and thus $d\left({ }^{*} x, x_{N}\right) \approx 0$, as the supremum is attained. From Theorem 7 we obtain $x_{n} \rightarrow x$ in $X$, ie $x_{n} \rightrightarrows x$ on $[0, T]$.

The final part follows directly from Proposition 1, applied to $X=(x, y)$, with $y=x^{\prime}$, $F(t, X)=(y, f(t)-h(x))$ and the fact that $F(t, X)$ is standard on $Q=\{x<0\}$.

\section{Behavior near the wall}

The aim of this Section is to describe the interaction of the oscillator with the wall. We show that we can distinguish three mutually exclusive scenarios, given by Propositions 13 , which are stable in terms of infinitesimal perturbations of the initial condition. It is also important that they are independent of the actual value of $L$, as long as it remains infinitely large.

The first scenario describes the (strict) bounce off the wall. We will need the usual variation of constants formula to write the solution for $x>0$ explicitly. 
Lemma 3 Let $t_{0}, t_{1} \in{ }^{*} \mathbb{R}$ be such that $\left[t_{0}, t_{1}\right] \subset{ }^{*}[0, T]$ and $x \geq 0$ on $\left[t_{0}, t_{1}\right]$. Then

$$
\begin{aligned}
x(t)= & x\left(t_{0}\right) \cos \sqrt{L}\left(t-t_{0}\right) \\
& +x^{\prime}\left(t_{0}\right) \frac{\sin \sqrt{L}\left(t-t_{0}\right)}{\sqrt{L}}+\int_{t_{0}}^{t} f(t-s) \frac{\sin \sqrt{L}\left(s-t_{0}\right)}{\sqrt{L}} d s \\
x^{\prime}(t)= & -x\left(t_{0}\right) \sqrt{L} \sin \sqrt{L}\left(t-t_{0}\right) \\
& +x^{\prime}\left(t_{0}\right) \cos \sqrt{L}\left(t-t_{0}\right)+\int_{t_{0}}^{t} f(t-s) \cos \sqrt{L}\left(s-t_{0}\right) d s
\end{aligned}
$$

for all $t \in\left[t_{0}, t_{1}\right]$.

Proof For $x \geq 0$, the equation reduces to $x^{\prime \prime}+L x=f(t)$, whence the formulas are obtained by variation of constants.

The proposition describing the bounce can be now stated as follows. Recall that $E(t)$ is the total energy of the system, defined by (7) above.

Proposition 2 Let $t_{0} \in{ }^{*}(0, T)$ be such that $x\left(t_{0}\right) \approx 0$ and $E\left(t_{0}\right) \gtrsim 0$. Then there exist $t_{\text {in }}<t_{\text {out }}$ infinitely close to $t_{0}$, such that $x\left(t_{\text {in }}\right)=x\left(t_{\text {out }}\right)=0, x>0$ in $\left(t_{\text {in }}, t_{\text {out }}\right)$ and $x^{\prime}\left(t_{\text {in }}\right) \approx-x^{\prime}\left(t_{\text {out }}\right)$ are not infinitesimal.

Proof Case 1. Assume first that $x\left(t_{0}\right)=0$ exactly, hence by assumption $x^{\prime}\left(t_{0}\right)=\tilde{v}_{0}$ is not infinitesimal. Let $\tilde{v}_{0}>0$ (the case $\tilde{v}_{0}<0$ being entirely symmetric). We set $t_{\text {in }}=t_{0}$. For simplicity of notation, let $t_{0}=0$. By Lemma 3 we have

$$
\begin{aligned}
x(t) & =\frac{\tilde{v}_{0}}{\sqrt{L}} \sin \sqrt{L} t+\frac{1}{\sqrt{L}} \int_{0}^{t} f(t-s) \sin \sqrt{L} s d s \\
x^{\prime}(t) & =\tilde{v}_{0} \cos \sqrt{L} t+\frac{1}{\sqrt{L}}\left(\int_{0}^{t} f^{\prime}(t-s) \sin \sqrt{L} s d s+f(0) \sin \sqrt{L} s\right)
\end{aligned}
$$

as long as $x(t) \geq 0$, which clearly holds on some nontrivial interval $[0, \delta)$. Let $t_{\text {out }}>0$ be smallest time such that $x\left(t_{\text {out }}\right)=0$. Observe that for $\tilde{t}=3 \pi / 2 \sqrt{L}$ the first term in (12) gives $-\tilde{v}_{0} / \sqrt{L}$, while the second is estimated by $c / L$, which would yield $x(\tilde{t})<0$, hence $t_{\text {out }}$ exists in the (infinitesimal) interval $(0,3 \pi / 2 \sqrt{L})$.

To locate $t_{\text {out }}$ more precisely, observe again from (12) that

$$
\left|\sin \sqrt{L} t_{\text {out }}\right| \leq \frac{c}{\sqrt{L}}
$$

On the other hand, for $\epsilon>0$ small enough, $|\sin y| \leq \epsilon$ is only possible if $|y-k \pi| \leq 2 \epsilon$ for some integer $k$. This imposes the condition

$$
\left|t_{\text {out }}-\frac{k \pi}{\sqrt{L}}\right| \leq \frac{c}{L}
$$


and in view of the above, $k=1$ is the only possibility. Hence we have

$$
\left|t_{\text {out }}-\frac{\pi}{2 \sqrt{L}}\right| \leq \frac{c}{L}
$$

and it follows from $(13)$ that $x^{\prime}\left(t_{\text {out }}\right) \approx-\tilde{v}_{0}$.

Case 2. If $x\left(t_{0}\right)<0$ with $x\left(t_{0}\right) \approx 0$ and $x^{\prime}\left(t_{0}\right)=\tilde{v}_{0}>0$ not infinitesimal, we start in the standard regime (cf. first part of Proposition 1) with both $x(t)$ and $x^{\prime}(t)$ being $S$-continuous. Whence clearly exists $t_{\text {in }}>t_{0}$, such that $t_{\text {in }} \approx t_{0}$, and $x\left(t_{\text {in }}\right)=0$, $x^{\prime}\left(t_{\text {in }}\right) \approx \tilde{v}_{0}$ and we continue as in case 1 .

Case 3. Assume finally that $x\left(t_{0}\right) \approx 0$ with $x\left(t_{0}\right)>0$, and $E\left(t_{0}\right)>0$ is not infinitesimal. Invoking again formulas from Lemma 3 , we deduce by an argument analogous to case 1 the existence of $t_{\text {in }}<t_{\text {out }}$ conforming to the assertion of the lemma. Note that in this case $t_{0} \in\left(t_{\text {in }}, t_{\text {out }}\right)$ and $x^{\prime}\left(t_{0}\right)$ might lie strictly between $x^{\prime}\left(t_{\text {in }}\right), x^{\prime}\left(t_{\text {out }}\right)$. On the other hand, we always have $\left|x^{\prime}\left(t_{\text {in }}\right)\right| \approx\left|x^{\prime}\left(t_{\text {out }}\right)\right| \approx \sqrt{2 E\left(t_{0}\right)}$.

It remains to consider the case $x\left(t_{0}\right) \approx 0, E\left(t_{0}\right) \approx 0$. We now employ the assumption (9), dealing first with zero force. In fact a somewhat weaker assumption on $f(t)$ would suffice.

Lemma 4 Let $x\left(t_{0}\right) \approx 0$ and $E\left(t_{0}\right) \approx 0$ and let $f(t)$ be an internal continuous function such that $f(t) \approx 0$ on $\left[t_{0}, t_{0}+\Delta\right]$. Then $E(t) \approx 0, x(t) \approx 0$ on $\left[t_{0}, t_{0}+\Delta\right]$.

Proof Multiplying the equation by $x^{\prime}$ yields $\frac{d}{d t} E(t) \approx 0$ and so $E(t) \approx 0$ follows by integration, whence obviously $x(t) \approx 0$ too.

If $f\left(t_{0}\right) \not \approx 0$, then either $f(t) \geq c>0$ or $f(t) \leq-c$ close enough to $t_{0}$, for some standard $c>0$. If $f\left(t_{0}\right) \approx 0$, then we can actually assume $f\left(t_{0}\right)=0$. Indeed, as $f(t)$ is a standard function, there is $\tilde{t}_{0} \approx t_{0}$ such that $f\left(\tilde{t}_{0}\right)=0$; clearly $x\left(\tilde{t}_{0}\right) \approx 0$ and $E\left(\tilde{t}_{0}\right) \approx 0$ holds. Having now set the case $f(t) \equiv 0$ aside, by the second part of (9), we have that $f^{\prime}\left(t_{0}\right)$ is a standard non-zero number. But this again means that on some right neighborhood of $t_{0}$, either $f(t)>0$ or $f(t)>0$ with the derivative close to $f_{+}^{\prime}\left(t_{0}\right)$. To summarize, we can assume that:

(14) there exist $\Delta>0$ and $c$ or $a$ standard positive such that one of the following holds on $\left[t_{0}, t_{0}+\Delta\right]$ :

(i) $f(t) \leq-c$

(ii) $f(t) \geq c$

(iii) $f\left(t_{0}\right)=0$ and $f^{\prime}(t) \leq-a$

(iv) $f\left(t_{0}\right)=0$ and $a \leq f^{\prime}(t) \leq \frac{5}{3} a$ 
Since we are now in a situation when the solution can switch between the regimes $x>0$ and $x<0$, that is to say between the equation $x^{\prime \prime}+L x=f(t)$ and $x^{\prime \prime}+h(x)=f(t)$, we will further simplify our considerations by showing that it is actually possible to set $h(x)=0$ without loss of generality.

Lemma 5 Let $t_{0}$ be such that $x\left(t_{0}\right) \approx 0, E\left(t_{0}\right) \approx 0$ and one of the cases in (14) holds on $\left[t_{0}, t_{0}+\Delta\right]$. Then for $x(t)<0$, the solution satisfies $x^{\prime \prime}=\tilde{f}(t)$, where $\tilde{f}(t)=f(t)-h(x(t))$ has the same property in (14) as $f(t)$ does, up to a possible adjustment of the constants $c, a$, or $\Delta>0$.

Proof By (8) above, Gronwall's lemma and the fact that $E\left(t_{0}\right) \approx 0$ we have $E(t) \leq$ $\mu+c\left(t-t_{0}\right)$, for some $\mu \approx 0$ and hence $h(x(t))$ and $\left(h(x(t))^{\prime}=h^{\prime}(x(t)) x^{\prime}(t)\right.$ can be estimated by a small standard $\varepsilon>0$. So after a possible adjustment of $c, a$ or $\Delta$, the variant of (14) holding for $f(t)$ holds for $\tilde{f}(t)$, too.

For the rest of this section, we will constantly make use of this lemma, by assuming that (1) has the simple form $x^{\prime \prime}=f(t)$ whenever $x(t)<0$.

We now observe that in cases (i) or (iii) of (14), the solution is pulled-out from the wall immediately.

Proposition 3 Let $t_{0} \in{ }^{*}[0, T)$ be such that $x\left(t_{0}\right)$ and $E\left(t_{0}\right) \approx 0$. Let $f(t)<0$ on $\left(t_{0}, t_{0}+\Delta\right]$. Then

$$
x(t) \approx \int_{t_{0}}^{t} F(s) d s, \quad t \in\left[t_{0}, t_{0}+\Delta\right]
$$

where $F(t)=\int_{t_{0}}^{t} f(s) d s$, and in particular, $x(t)<0$ whenever $t \gtrsim t_{0}$.

Proof If $x\left(t_{0}\right) \leq 0$, then (15) follows simply by integration. If $x\left(t_{0}\right)>0$, we again employ the formula (11). It follows that no later than at $t=t_{0}+\pi / \sqrt{L}$, the first two terms are negative, to which the second term also gives a negative (albeit infinitesimal) contribution. Hence, there is $t_{\text {out }} \in\left(t_{0}, t_{0}+\pi / \sqrt{L}\right)$ such that $x\left(t_{\text {out }}\right)=0$, and clearly $x^{\prime}\left(t_{\text {out }}\right)<0$, with $x^{\prime}\left(t_{\text {out }}\right) \approx 0$. We are thus reduced to a previous case with $t_{\text {out }}=t_{0}$.

It remains to deal with the most interesting case, namely that $f(t) \geq 0$ acting on a system for which $x\left(t_{0}\right)$ and $E\left(t_{0}\right) \approx 0$. The problem is that we cannot say a priori where the solution will be. Indeed the situation $x(t)>0$ and $x(t)<0$ can change infinitely many times, switching between the equation $x^{\prime \prime}=f(t)$ and $x^{\prime \prime}+L x=f(t)$. We however do observe the following. 
Lemma 6 Let $f(t)$ satisfy (14). Then the set of zero times $\left\{t \in\left[t_{0}, t_{0}+\Delta\right] ; x(t)=0\right\}$ is an internal hyperfinite set.

Proof This is an easy application of the transfer principle. Assume that $L>0$ in (6) is a standard number. Let $x(t)$ be an arbitrary solution. If $x\left(t_{0}\right)=0$, then either $x^{\prime}\left(t_{0}\right) \neq 0$, or, if $x^{\prime}\left(t_{0}\right)=0$, we invoke the equation and (9) to show that $x^{\prime}(t) \neq 0$ on some $\mathcal{P}\left(t_{0}, \delta\right)$. It follows that each zero point is isolated, hence the set of zero points in $[0, T]$ is finite.

By transferring a suitable formalization of the last assertion, it follows that for arbitrary hyperreal $L>0$, the set of zero points is hyperfinite.

Our strategy is as follows: by the previous lemma, the zero points of $x(t)$ in $\left[t_{0}, t_{0}+\Delta\right]$ can be denoted by $t_{i}, i=1, \ldots N$ with some $N \in \mathbb{N}^{\infty}$. Set $E_{i}=E\left(t_{i}\right)$. We will prove recursive estimates of $E_{i+1}$ in terms of $E_{i}$, and the result is then obtained by "integration" of these local estimates into a global one which would entail $E_{i} \approx 0$ for all $i \leq N$. It then follows that $x(t) \approx 0$ and $x^{\prime}(t) \approx 0$ on $\left[t_{0}, t_{0}+\Delta\right]$.

Let us start with the intervals where $x>0$.

Lemma 7 Let $x(t)>0$ on $\left(t_{i}, t_{i+1}\right)$.

1. There holds

$$
E_{i+1}-E_{i} \leq \mu\left(t_{i+1}-t_{i}\right)
$$

where $\mu \approx 0$ only depends on $f(t)$.

2. Moreover, if $f(t)$ is nondecreasing, then even

$$
E_{i+1}-E_{i} \leq 0
$$

Proof We write

$$
E\left(t_{i+1}\right)-E\left(t_{i}\right)=\int_{t_{i}}^{t_{i+1}} x^{\prime}(t) f(t)=[x(t) f(t)]_{t=t_{i}}^{t=t_{i+1}}-\int_{t_{i}}^{t_{i+1}} x(t) f^{\prime}(t) d t
$$

The increment is zero since $x\left(t_{i}\right)=x\left(t_{i+1}\right)=0$. By the boundedness of the energy (cf Lemma 1) we have $|x(t)| \leq c / L^{1 / 2}$. Hence the integrand is estimated by an infinitesimal constant and (16) follows. Moreover, if $f^{\prime}(t) \geq 0$, the integrand is non-positive and we obtain (17).

The situation for $x<0$ is more delicate and it will require several different estimates. It is also more convenient to work with the velocities $v_{i}=x^{\prime}\left(t_{i}\right)$. Recall that $c>0$ is a standard positive constant, the value of which can change from line to line in the inequalities appearing below. 
Lemma 8 Let and $x<0$ and $f(t) \geq 0$ on $\left(t_{i}, t_{i+1}\right)$.

1. If $f(t)$ is nonincreasing, then

$$
\left|v_{i+1}\right|-\left|v_{i}\right| \leq 0
$$

2. If $f(t) \geq c>0$, then

$$
\left|v_{i+1}\right|-\left|v_{i}\right| \leq c\left|v_{i}\right|\left(t_{i+1}-t_{i}\right)
$$

3. If $f^{\prime}(t) \geq a>0$, then

$$
\left|v_{i+1}\right|-\left|v_{i}\right| \leq c\left|v_{i}\right|^{1 / 2}\left(t_{i+1}-t_{i}\right)
$$

4. Finally, if $0<a \leq f^{\prime}(t) \leq 5 a / 3$ and $f\left(t_{0}\right)=0$ holds, then

$$
\left|v_{i+1}\right|-\left|v_{i}\right| \leq \frac{\left|v_{i}\right|}{t_{i}}\left(t_{i+1}-t_{i}\right)
$$

for any $i \geq 1$.

Proof 1. If $f(t) \geq 0$ is nonincreasing, then $f^{\prime}(t) \leq 0$ and we deduce that $E_{i+1} \leq E_{i}$ as in second part of Lemma 7, and since $x\left(t_{i}\right)=x\left(t_{i+1}\right)=0$, we obtain $\left|v_{i+1}\right| \leq\left|v_{i}\right|$.

For the rest of the proof, we shift to $t_{i}=0$ and $t_{i+1}=t_{1}$ in order to simplify the notation. Recall that in virtue of Lemma 5 , we work with the equation

$$
x^{\prime \prime}=f(t)
$$

Writing $F(t)=\int_{0}^{t} f(s) d s$, we have

$$
\begin{aligned}
x^{\prime}(t) & =v_{0}+F(t) \\
x(t) & =t v_{0}+\int_{0}^{t} F(s) d s
\end{aligned}
$$

and the return time $t_{1}$ is given by the condition

$$
-v_{0}=\frac{1}{t_{1}} \int_{0}^{t_{1}} F(s) d s
$$

Our aim is to estimate

$$
\left|v_{1}\right|-\left|v_{0}\right|=x^{\prime}\left(t_{1}\right)+v_{0}=2 v_{0}+F\left(t_{1}\right)=\frac{1}{t_{1}} \int_{0}^{t_{1}} F\left(t_{1}\right)-2 F(s) d s
$$

In the remaining estimates, we use the Taylor expansion:

$$
F(t)=F(0)+t F(0)+\frac{1}{2} t^{2} F^{\prime}(\tau)=t f(0)+\frac{1}{2} t^{2} f^{\prime}(\tau)
$$

for some $\tau \in(0, t)$. 
2. In particular, (22) implies $|F(t)-t f(0)| \leq c t^{2}$, which gives

$$
\frac{1}{t_{1}} \int_{0}^{t_{1}} F\left(t_{1}\right)-2 F(s) d s \leq \frac{1}{t_{1}} \int_{0}^{t_{1}} t_{1} f(0)-2 s f(0)+c t_{1}^{2} d s \leq c t_{1}^{2}
$$

On the other hand, for $f(t) \geq c>0$, we have $F(t) \geq c t$ and from (20) one obtains

$$
t_{1} \leq c\left|v_{0}\right|
$$

Combining with (23) yields the conclusion.

3. For $f^{\prime}(t) \geq a>0$, we have $f(t) \geq a t$ and thus $F(t) \geq c t^{2}$. From (20) we deduce

$$
t_{1} \leq c\left|v_{0}\right|^{1 / 2}
$$

and again use (23) to arrive at the conclusion.

4. We again employ (22), recalling that $a \leq f^{\prime}(\tau) \leq 5 a / 3$. Consequently

$$
\frac{1}{t_{1}} \int_{0}^{t_{1}} F\left(t_{1}\right)-2 F(s) d s \leq \frac{1}{t_{1}} \int_{0}^{t_{1}} t_{1} f(0)+\frac{5}{6} t_{1}^{2} a-2 s f(0)-s^{2} a d s=\frac{1}{2} a t_{1}^{2}
$$

On the other hand, we have $F(t) \geq f(0) t$, hence by (20)

$$
\left|v_{0}\right| \geq \frac{1}{2} f(0) t_{1}
$$

Combining (24), (25) with (21) gives

$$
\left|v_{1}\right|-\left|v_{0}\right| \leq \frac{a\left|v_{0}\right|}{f(0)} t_{1}
$$

Shifting back to original time, $t_{1}$ becomes $t_{i+1}-t_{i}$ and $f(0)$ becomes $f\left(t_{i}\right)$. But $f\left(t_{i}\right) \geq a t_{i}$ by our assumptions on $f(t)$ and we are done.

The synthesis of the above difference estimates is completed in the following key result.

Proposition 4 Let $x\left(t_{0}\right) \approx 0, E\left(t_{0}\right) \approx 0$. Let $f(t) \geq 0$ on $\left[t_{0}, t_{0}+\Delta\right]$. Then $E(t) \approx 0$ on $\left[t_{0}, t_{0}+\Delta\right]$.

Proof We will show that $E_{i} \approx 0$ for all $i=0, \ldots, N$. It is easy to observe that $E_{i} \approx 0$ implies $E(t) \approx 0$ for all $t \in\left[t_{i}, t_{i+1}\right]$. In view of (14), we only need to deal with two cases.

1. Let $f(t) \geq c>0$. If $x(t)>0$ on $\left(t_{i}, t_{i+1}\right)$, by Lemma 7 , part 1 , we have

$$
E_{i+1}-E_{i} \leq \mu\left(t_{i+1}-t_{i}\right)
$$


with some positive $\mu \approx 0$. If, on the other hand, $x(t)<0$, we have $\left|v_{i+1}\right|-\left|v_{i}\right| \leq$ $c\left|v_{i}\right|\left(t_{i+1}-t_{i}\right)$ with some standard $c>0$. Noting that $E_{i}=\frac{1}{2}\left|v_{i}\right|^{2}$, it is easy to deduce

$$
E_{i+1}-E_{i} \leq c E_{i}\left(t_{i+1}-t_{i}\right)
$$

with possibly different $c>0$. Combining the both we always have

$$
E_{i+1}-E_{i} \leq\left(k E_{i}+\mu\right)\left(t_{i+1}-t_{i}\right)
$$

for some standard $k>0$ and infinitesimal $\mu>0$. If follows by Lemma 9 that $E_{i} \leq\left(E_{0}+\mu / k\right) \exp \left(k\left(t_{i}-t_{0}\right)\right)$. Hence if $E_{0} \approx 0$, we have $E_{i} \approx 0$ for all $i$.

2. Let $f\left(t_{0}\right)=0$ and $a \leq f^{\prime}(t) \leq \frac{5}{3} a$ on $\left[t_{0}, t_{0}+\Delta\right]$, where $a>0$ is standard. We can again set $t_{0}=0$ for simplicity.

On intervals where $x(t)<0$, we have two estimates at our disposal, namely (18) and (19) from part 3 and 4 of Lemma 8.

On intervals where $x(t)>0$, since $f(t)$ is increasing, $E_{i+1}-E_{i} \leq 0$ by part 2 of Lemma 7, and so $\left|v_{i+1}\right|-\left|v_{i}\right| \leq 0$, which means that (18) and (19) actually hold for all $i$.

One first employs (18). By Lemma 11 with $g(u)=u^{1 / 2}$ and $f(t)=1$, we obtain $\left|v_{i}\right|^{1 / 2} \leq\left|v_{0}\right|^{1 / 2}+c t_{i}$ and hence

$$
\left|v_{i}\right| \leq c\left(t_{i}^{2}+\left|v_{0}\right|\right)
$$

Therefore, one can choose $t_{\iota}>0$ infinitesimal so that $\left|v_{\iota}\right| / t_{\iota} \approx 0$. By Lemma 10 and (19), the values of $z_{i}=\left|v_{i}\right| / t_{i}$ are nonincreasing, and thus $\left|v_{i}\right| / t_{i} \leq\left|v_{\iota}\right| / t_{\iota} \approx 0$ for all $i \geq \iota$. Hence $\left|v_{i}\right| \approx 0$ for all $i$ and we are done.

\section{Proof of Theorem 2}

Set

$$
\begin{aligned}
J & =\operatorname{st}\{t \in I ; x \approx \tilde{x} \text { on }[0, t]\} \\
t_{0} & =\sup J
\end{aligned}
$$

Note that the supremum is taken in $\mathbb{R}$ (not in ${ }^{*} \mathbb{R}$ ) here. Clearly $J \subset \mathbb{R}$ is an interval. Since by Lemma 1 the energy is bounded and thus $x(t)-\tilde{x}(t)$ is (standard) Lipschitz, one sees that $J$ is closed, ie $I=\left[0, t_{0}\right]$ for some $t_{0} \in[0, T]$. We will show that $t_{0}<T$ leads to contradiction by a variant of the usual creeping argument. 
Observe first that $x(t)$ and $\tilde{x}(t)<0$ on some $[0, \Delta]$ by continuity, and thus remain infinitely close by Lemma 2 . This means that $t_{0}>0$, and we track down for repeated future use that in particular

$$
x(t) \approx \tilde{x}(t) \quad \text { on }\left(t_{0}-\Delta_{0}, t_{0}\right] \text { for some } \Delta_{0}>0 \text { standard }
$$

1. Let $x\left(t_{0}\right)<0$ and $x\left(t_{0}\right) \not \approx 0$. Then in addition to (27) we have $x(t), \tilde{x}(t)<0$ and thus also $x^{\prime}(t) \approx \tilde{x}^{\prime}(t)$ on $\left(t_{0}-\Delta_{0}, t_{0}\right]$. In particular it follows that $x^{\prime}\left(t_{0}\right) \approx \tilde{x}^{\prime}\left(t_{0}\right)$ and by Lemma 2 we deduce $x(t) \approx \tilde{x}(t)$ also for $t \in\left[t_{0}, t_{0}+\Delta\right)$ with some standard $\Delta>0$, which contradicts the definition of $t_{0}$.

2. It remains to discuss the situation where $x\left(t_{0}\right) \approx 0$. We further distinguish the following sub-cases:

2a. Let $E\left(t_{0}\right) \not \approx 0$. Then we can apply Proposition 2 to $x(t)$ and obtain $t_{\text {in }}<t_{\text {out }}$ infinitely close to $t_{0}$. Now $x^{\prime}\left(t_{\text {in }}\right) \gtrsim 0$ and $x\left(t_{\text {in }}\right)=0$, so we can also assume that $x(t)<0$ on $\left(t_{i n}-\Delta_{0}, t_{i n}\right)$. It now follows from (27) and Proposition 1 that the second solution $\tilde{x}(t)$ will satisfy $\tilde{x}\left(\tilde{t}_{i n}\right)=0$ for some $\tilde{t}_{i n} \approx t_{i n} \approx t_{0}$ with $\tilde{x}\left(\tilde{t}_{i n}\right) \approx x^{\prime}\left(t_{i n}\right)$; in particular, Proposition 2 applies to $\tilde{x}(t)$ as well.

Hence also $\tilde{x}^{\prime}\left(\tilde{t}_{\text {out }}\right) \approx x^{\prime}\left(t_{\text {out }}\right)$, which implies $x(t) \approx \tilde{x}(t)$ on some standard $\left[t_{0}, t_{0}+\Delta\right)$. This contradicts (26).

2b. Let $x\left(t_{0}\right) \approx 0$ and also $E\left(t_{0}\right) \approx 0$. We also have $\tilde{x}\left(t_{0}\right) \approx 0$. We also necessarily have $\tilde{E}\left(t_{0}\right) \approx 0$; for otherwise we argue as in 2 a. We now invoke the structural assumption (9). If $f(t) \equiv 0$ on some $\left[t_{0}, t_{0}+\Delta\right]$, then both solutions remain infinitely close to 0 and hence to each other by Lemma 4 .

Otherwise, we can assume that (14) applies. Either by Proposition 3 both solutions are pulled-out and keep infinitely close in virtue of (15), or by Proposition 4 both solutions remain infinitely close to $x=0$ up to some $t_{0}+\Delta$. At any rate, this again contradicts the definition of $t_{0}$ and the proof is complete.

\section{Appendix}

Here we prove three auxiliary lemmas on "integration" of certain difference equations. The results are proven by induction for standard sequences; hence by transfer they hold for internal hyper-finite sequences as required.

The first lemma is just a discrete version of Gronwall's lemma. 
Lemma 9 Let $E_{i} \geq 0$ satisfy

$$
E_{i+1}-E_{i} \leq\left(k E_{i}+\mu\right)\left(t_{i+1}-t_{i}\right), \quad \forall i=0, \ldots, N
$$

where $k \geq 0, \mu>0$. Then

$$
E_{i} \leq E_{0} \exp \left(k\left(t_{i}-t_{0}\right)\right)+\frac{\mu}{k}\left(\exp \left(k\left(t_{i}-t_{0}\right)\right)-1\right), \quad \forall i=0, \ldots, N
$$

Proof Set $F_{i}=k E_{i}+\mu$. Then $F_{i}>0$ and (28) can be can be rewritten as

$$
\frac{F_{i+1}-F_{i}}{F_{i}} \leq k\left(t_{i+1}-t_{i}\right)
$$

Furthermore,

$$
\ln F_{i+1}-\ln F_{i}=\ln \frac{F_{i+1}}{F_{i}}=\ln \left(1+\frac{F_{i+1}}{F_{i}}-1\right) \leq \frac{F_{i+1}}{F_{i}}-1=\frac{F_{i+1}-F_{i}}{F_{i}}
$$

by an elementary inequality $\ln (1+x) \leq x$, which is true for any $x>-1$. Thus we have $\ln F_{i+1}-\ln F_{i} \leq k\left(t_{i+1}-t_{i}\right)$. Summing up and exponentiating we obtain

$$
F_{i} \leq F_{i} \exp \left(k\left(t_{i}-t_{0}\right)\right)
$$

which can be rewritten as the desired conclusion (29).

Second lemma deals with homogeneous equations.

Lemma 10 Let $u_{i}$ satisfy

$$
u_{i+1}-u_{i}=f\left(t_{i}, u_{i}\right)\left(t_{i+1}-t_{i}\right), \quad \forall i=0, \ldots, N
$$

where $f=f(t, u)$ is 0-homogeneous: for any $\lambda>0$, one has $f(\lambda t, \lambda u)=f(t, u)$. Set $u_{i}=t_{i} z_{i}$. Then $z_{i}$ satisfy

$$
\left(z_{i+1}-z_{i}\right) t_{i+1}=\left(f\left(1, z_{i}\right)-z_{i}\right)\left(t_{i+1}-t_{i}\right)
$$

Proof One writes

$$
u_{i+1}-u_{i}=t_{i+1} z_{i+1}-t_{i} z_{i}=\left(z_{i+1}-z_{i}\right) t_{i+1}+z_{i}\left(t_{i+1}-t_{i}\right)
$$

Further, the homogeneity gives $f\left(t_{i}, u_{i}\right)=f\left(1, z_{i}\right)$ and (31) follows at once from (30).

The last lemma corresponds to integration of separable equations. 
Lemma 11 Let $u_{i}$ satisfy

$$
u_{i+1}-u_{i} \leq g\left(u_{i}\right) f\left(t_{i}\right)\left(t_{i+1}-t_{i}\right), \quad \forall i=0, \ldots, N
$$

with $g(u), f(t)$ nondecreasing and $g(u)>0$. Then

$$
G\left(u_{i}\right)-G\left(u_{0}\right) \leq F\left(t_{i}\right)-F\left(t_{0}\right), \quad \forall i=0, \ldots, N
$$

where $G(u), F(t)$ are primitive functions to $1 / g(u)$ and $f(t)$, respectively.

Proof Dividing by $g\left(u_{i}\right)>0$ preserves the inequality. On the left hand side, we employ the mean value theorem to estimate

$$
\frac{u_{i+1}-u_{i}}{g\left(u_{i}\right)}=G^{\prime}\left(u_{i}\right)\left(u_{i+1}-u_{i}\right) \geq G^{\prime}(\eta)\left(u_{i+1}-u_{i}\right)=G\left(u_{i+1}\right)-G\left(u_{i}\right)
$$

where $\eta$ is between $u_{i}$ and $u_{i+1}$. Likewise the right hand side is estimated through

$$
f\left(t_{i}\right)\left(t_{i+1}-t_{i}\right) \leq F^{\prime}(\tau)\left(t_{i+1}-t_{i}\right)=F\left(t_{i+1}\right)-F\left(t_{i}\right)
$$

with $\tau$ between $t_{i}$ and $t_{i+1}$. Combining and summing up the estimates, we arrive at (32).

\section{Acknowledgments}

Jakub Slavík was supported by the grant SVV-2016-260335.

\section{References}

[1] V Benci, M Forti, M Di Nasso, The eightfold path to nonstandard analysis, from: "Nonstandard methods and applications in mathematics", Lect. Notes Log. 25, Assoc. Symbol. Logic, La Jolla, CA (2006) 3-44

[2] A F Filippov, Differential equations with discontinuous righthand sides, volume 18 of Mathematics and its Applications (Soviet Series), Kluwer Academic Publishers Group, Dordrecht (1988); doi: 10.1007/978-94-015-7793-9

[3] A E Hurd, P A Loeb, An introduction to nonstandard real analysis, volume 118 of Pure and Applied Mathematics, Academic Press, Inc., Orlando, FL (1985)

[4] M Kunze, Non-smooth dynamical systems, volume 1744 of Lecture Notes in Mathematics, Springer-Verlag, Berlin (2000); doi: 10.1007/BFb0103843

[5] M Kunze, R Ortega, Complete orbits for twist maps on the plane: extensions and applications, J. Dynam. Differential Equations 23 (2011) 405-423; doi: 10.1007/s10884010-9185-y 
[6] M Kunze, R Ortega, Complete orbits for twist maps on the plane: the case of small twist, Ergodic Theory Dynam. Systems 31 (2011) 1471-1498; doi: $10.1017 / \mathrm{S} 0143385710000520$

[7] S Marò, Coexistence of bounded and unbounded motions in a bouncing ball model, Nonlinearity 26 (2013) 1439-1448; doi: 10.1088/0951-7715/26/5/1439

[8] E Nelson, Internal set theory: a new approach to nonstandard analysis, Bull. Amer. Math. Soc. 83 (1977) 1165-1198; doi: 10.1090/S0002-9904-1977-14398-X

[9] K Rajagopal, A generalized framework for studying the vibrations of lumped parameter systems, Mechanics Research Communications 37 (2010) 463-466; doi: 10.1016/j.mechrescom.2010.05.010

[10] A Robinson, Non-standard Analysis, Princeton landmarks in mathematics and physics, Princeton University Press (1974)

[11] M Väth, Nonstandard analysis, Birkhäuser Verlag, Basel (2007); doi: 10.1007/978-37643-7774-8

[12] Z Yuan, V Průša, K Rajagopal, A Srinivasa, Vibrations of a lumped parameter massspring-dashpot system wherein the spring is described by a non-invertible elongationforce constitutive function, International Journal of Non-Linear Mechanics 76 (2015) 154-163; doi: 10.1016/j.ijnonlinmec.2015.06.009

Department of Mathematical Analysis, Charles University, Sokolovská 83, 18675 Praha 8, Czech Republic

Department of Mechanical Engineering, Texas A\&M University, College Station, TX 77843 USA

Department of Mathematical Analysis, Charles University, Sokolovská 83, 18675 Praha 8, Czech Republic

prazak@karlin.mff.cuni.cz, krajagopal@tamu.edu, slavikj@karlin.mff.cuni.cz

Received: 7 April 2016 Revised: 9 March 2017 